one must be an odd number while that of the other is twice this odd number when $s_{1} s_{2}=s_{2}^{2} s_{1}^{2}$; but when $s_{1} s_{2}=s_{2}^{-2} s_{1}^{-2}$ it is only necessary that the order of one is three times that of the other.

UNIVERSITY OF ILLINOIS.

\title{
THE TEACHING OF MECHANICS.
}

An Elementary Treatise on Theoretical Mechanics. By J. H. Jeans. Ginn and Company, 1907. viii +364 pp.

There are few topics in elementary mathematics that are more generally mishandled by the writers of our text books than Newton's three laws of motion. Perhaps it would be more accurate to say that the applications of the laws are generally misunderstood and that consequently the exponents of axioms which form the foundation of the mathematical science of mechanics rarely fail to makie some fundamental error which destroys at the outset any hope of a logical development of the subject. The worst cases generally occur in the books which are published under the title "Physics." It frequently happens that the authors have not mastered the meaning of the laws; more frequently they show a want of care in their statements and explanations. In either case the effect on the student must be the same - a nebulous conception of the whole subject and a general impression that one can get along perfectly well in physics or engineering without bothering to understand what facts are directly observed and which can be deduced from the laws of motion. A man who wishes to rise to the higher levels of these professions must know such matters.

Perhaps it will not be altogether out of place to insist here on some points in the teaching in this country of applied mathematics or mathematical physics, whatever be the name we like to give to the science which concerns itself with the application of mathematics to problems in which space, time, and matter are supposed to be related by certain definitely stated laws. At the outset, the subject is a "pure" science in exactly the same way that pure mathematics is so, in that it rests solely on definitions and axioms which have no necessary relation to the phenomena of nature. Every problem attacked is an ideal, not an actual problem. The statement of the ideal problem must conform to the laws laid down if it is to fall within the 
province of the subject. In order that a real physical problem may be treated by the science, it is necessary that the laws of the science should be the same as the laws which govern the phenomena of nature. Here the "application" comes in. We frame the laws of the science in such a manner that we may imitate as far as possible the laws which are supposed to govern natural phenomena. In this way we may logically develop the subject and, at the same time, coordinate our experiences of nature. If we find that the conclusions of an ideal problem, correctly worked out, differ from the solution furnished by experiment, we must, if the "application" is to be maintained, alter the laws of our science. The ideal solution was correct but the "application" was wrong.

As matters now stand, we have therefore three subjects to consider : pure mathematics, physics (neither of which it is necessary to define here) and that ideal science for which I shall use the English term, applied mathematics. In the United States applied mathematics has been almost entirely treated as a department of physics. It has not been considered as a science in itself, but only in so far as it was useful to physics. The backwardness of its development here would seem to be largely due to this view. It has usually been taught by the experimental physicist, and the pure mathematicians have neglected it. And yet, as a science based on exact definitions and axioms, the pure mathematician is better equipped to teach its elements than the physicist, who is obliged to concentrate much of his attention on natural phenomena. However this may be, some reconstruction of our methods is necessary if we are to have our share in the developments which are taking place elsewhere. We cannot hope immediately to reach the ideal condition in which every worker in physics has received a thorough training in applied mathematics, but we can recognize the defects of the present system and make a start towards their elimination.

Professor Jeans has grappled with the difficulty in his book. It has hitherto been expected that in a single course on statics and dynamics the student should be led from the elements of the subject, at least as far as the applications of Lagrange's equations, and this without a very extended knowledge of the calculus. With the average student, whether his main study be physics or mathematics, this is scarcely possible. In most of our colleges and universities we recognize the necessity of 
first and second courses in calculus and one can hardly hope for equal efficiency in the study of mechanics without a similar method of procedure. If the school courses in physics were replaced by courses in elementary mechanics - the foundation of all physical theories - or if the same courses were laid out more with regard to the theoretical than to the experimental side, it would not be difficult to give the student a sufficient grasp of mechanics in a succeeding year's work. As a matter of fact, the experiments in a first course on mechanics can be almost entirely replaced by illustrations drawn from the ordinary phenomena of daily life in which every boy takes an interest, and he would, learn much more surely the meaning and application of the laws of the subject than by using or looking at carefully constructed pieces of apparatus designed to illustrate special points. As long as the elements of mechanics are simply considered as an adjunct of physics and not as the basis of a science governed by exact laws, so long will there be an absence of any real development of applied mathematics. There are not short cuts to the higher branches of these subjects any more than there are to those of pure mathematics or of any other progressive science. The principles must not only be understood but thoroughly absorbed and used by the student for the solution of problems. As suggested above, this might be effected, partially at least, by inserting amongst the college entrance papers one on mechanics as the best introduction to the college courses in physics, the treatment being similar to that given in the numerous elementary text-books on statics and dynamics used in the English schools.

We have however, to deal with conditions as they are, and to consider how far Professor Jeans's book goes towards meeting them. It starts with the elements - kinematics and the laws of motion - and closes with the motion of a top, Euler's and Lagrange's equations, and Hamilton's principle. The main portion is undoubtedly a success in giving as rapid a development as can be expected. But in Chapters XI, XII the transition to the motion of a rigid body and to abstract dynamics is made with a jump which can hardly fail to leave the student behind. One receives the impression that these chapters have been inserted to satisfy the demands of the physicists, since their higher courses require the use of Lagrange's equations and generalized coordinates.

To come to details, the first chapter on kinematics is made a 
little hard for the beginner owing to the methods by which vectors and centroids are introduced; the mathematical parts of these might have been omitted or left until a later stage. In the second chapter, the laws of motion are clearly and accurately stated, and the deduction of the fundamental equation of dynamics

$$
P=m f
$$

(where $P$ is the force which produces in a mass $m$ an acceleration $f$ ) is made in such a manner as to show its importance for the whole subject. In the following chapter the experimental laws of extensibility of a string and of friction between two surfaces are stated, so that materials are at hand for the solution of many problems which bear a close relation to those found in nature. The selection of these problems is well made. The author has avoided the insertion of many examples in which the difficulties are chiefly mathematical, although he does not forget that the student has to be taught the application of mathematics to physical problems. One is only too familiar with devices for avoiding the use of mathematics - the laziness which takes the most pains.

Chapter V is somewhat difficult and might have been expanded, without adding to the length of the book, by cutting out some of the numerous determinations of centers of gravity given in the following chapter. The applications to problems of couples and forces in three-dimensional space are not easy to teach, while the idea of center of gravity is simple and is generally possessed by the student beforehand. One of the best expositions is that on work and energy, leading to a discussion of stability.

In the problems illustrating the motion of a particle under special forces, it is useful to find that motion under gravity is fully treated and that central forces, interesting as they are for the astronomer and the mathematician, are only given in sufficient detail to show how the laws of motion and that of gravitation are applied to the motions of the planets. Some advantage might have been gained by placing together the three principal methods of expressing the velocities and accelerations of a particle moving in one plane.

Fifteen years of attempts by the writer of this review to cover ground similar to that given in the first three hundred pages of this volume, with various text-books, occasionally 
without a text-book, and finally one year with Professor Jeans's book, convey a strong impression that the last, so far as he knows, is the best adapted to the needs of the American student under present conditions. That constant assistance and explanation will be required from the instructor is no defect; these are expected in the class-room, especially in a subject where the best results can only be obtained by outside work on the part of the student in the solution of problems and subsequent demonstration by the instructor. The few criticisms made here are on points not essential for the successful use of the book. Accuracy of statement is one of its chief and most pleasing characteristics. It is also well printed, and there is an absence of the too numerous varieties of type, designed like the headlines of a yellow journal to draw attention, which disfigure so many modern text-books.

The author is to be congratulated on a real advance towards the solution of the problem of introducing a first course in the teaching of mathematical physics in American colleges and universities.

YaLe University.

Ernest W. Brown.

\section{ECONOMICS.}

The Nature of Capital and Income. By Irving Fisher. New York, The Macmillan Company, 1906. xxi $+427 \mathrm{pp.}$ The Rate of Interest. By Irving Fisher. New York, The Macmillan Company, 1907. xxii $+422 \mathrm{pp}$.

ONE might almost say that, directly or indirectly, consciously or unconsciously, everybody is forced into intimate and vital relation with a large portion of the body of facts with which economics deals, and hence one might infer that the study of economics in one way or another cannot fail to be of interest to most rational beings. As an art, economics is of hoary antiquity ; as a domain of thought, recognized, delimited, and dignified with a special name, it is relatively modern; as a science, it is but in its infancy. Strictly speaking, it comes into close relation with mathematics only as it becomes a system of deduction; and in so far as it may have advanced to the deductive stage, it is mathematics. Of the authors who have materially contributed to the inclusion of their chosen science of economics 